\title{
A Micromachined 2DOF Nanopositioner with Integrated Capacitive Displacement Sensor
}

\author{
Lujun Ji, Yong Zhu, S. O. Reza Moheimani, Mehmet Rasit Yuce \\ School of Electrical Engineering and Computer Science, the University of Newcastle \\ University Drive, Callaghan, NSW, 2308, Australia \\ Email: \{lujun.ji, yong.zhu, reza.moheimani, mehmet.yuce\}@newcastle.edu.au
}

\begin{abstract}
This paper presents the design, fabrication and characterization of a micromachined two degrees-of-freedom (2DOF) nanopositioner. The proposed micro-electro-mechanical system (MEMS) stage, consisting of comb-drive actuators and on-chip capacitive displacement sensors in both $X$ and $Y$ directions, can simultaneously actuate the microstage and sense the corresponding displacements. A commercial capacitive readout IC (MS3110) is used for the open-loop capacitive sensing. The first resonance frequency of the stage is measured to be $4.24 \mathrm{kHz}$. The positioner has a dynamic range from $\mathbf{- 6 . 2 7}$ $\mu \mathrm{m}$ to $+6.64 \mu \mathrm{m}$ at an actuation voltage of $100 \mathrm{~V}$.
\end{abstract}

\section{INTRODUCTION}

With the development of the MEMS technology, MEMSbased positioning stages have attracted more and more attention in numerous applications in micro-/nano-scale positioning and manipulation systems due to their small size, low cost, fast response, and flexibility for system integration. The applications, such as microlens-array based optical cross connect $(\mathrm{OXC})$ [1], micro-confocal imaging [2], scanning probe microscopy (SPM)-based high-density data storage [3], and optical and magnetic pickup heads [4][5], demand the capability of positioning over a motion rage of micrometers and with a resolution of nanometers.

Piezoelectric stages have been widely used for nanopositioning applications [6]-[9]. SPM is a typical example, wherein piezoactuators are used for $X$-, $Y$-, and $Z$-positioning. Although piezoactuators are capable of providing nanometer resolutions, the inherent hysteresis and creep that are characteristics of piezoelectric material can cause significant open-loop positioning errors. Thus, piezoactuators require sophisticated nonlinear compensation techniques [10]-[11]. In addition, the relatively large sizes of most commercially available piezoelectric stages (usually around $10 \mathrm{~cm}$ ) limit their use in micro-scale system integration. MEMS-based positioning stages can offer a good alternative to piezoelectric stages due to their small size, high resonance frequency, precise positioning control, and flexibility in system integration.

Liu et al. have reported on the design, fabrication and testing of a MEMS-based 3-axis positioning stage [12]. Inplane and out-of-plane electrostatic actuators (comb-drive and

This research is funded by Australian Research Council (ARC) discovery grant - DP0774287. parallel plate) are employed to drive the stage to move independently along the $X Y Z$ directions. The drawback of this design is that there is no sensing implemented for closed-loop feedback control to achieve important objectives such as improved dynamic behaviour of the actuator with fast response time, precise position control and continuous tuning of position [6].

To provide functional improvement to [12], this paper presents a MEMS-based 2DOF nanopositioning stage, which utilizes four sets of electrostatic linear comb drives for jointly driving the stage to produce motions in the $X$ and $Y$ directions, and four sets of integrated comb-drive displacement sensors in conjunction with a commercial capacitive readout IC (MS3110) for simultaneously reading out the corresponding displacements.

\section{DEVICE DESIGN}

The schematic diagram of the MEMS-based 2DOF nanopositioner is depicted in Fig. 1. On the silicon layer, distributed around the center stage, four sets of in-plane combdrive actuators are employed to drive the center stage along the $X$ and $Y$ directions, with each actuator set consisting of four banks of combs. And, four sets of comb-drive sensors are designed to sense the corresponding displacements of the center stage, with each sensor set consisting of two banks of combs. Four tethering beams are used to suspend the center stage and transmit in-plane motions from the comb-drive actuators.

To minimize the cross-coupling of motions among different directions, orthogonal configuration of the $X Y$ actuators is chosen [12]. For instance, when the actuators (I) and (II) drive the center stage in the $X$ direction, the two tethering beams in the $X$ direction are tensile, thus no displacements in the $Y$ direction are generated. The $X$ directional actuation forces not only deflect the two tethering beams in the $Y$ direction but also introduce $X$-directional loads to the suspension beams of actuators (III) and (IV). In order not to interfere with $Y$-directional positioning of the stage, the suspension beams of actuators (III) and (IV) must have a high lateral stiffness in the $X$ direction to minimize $X$-directional displacements of the movable comb fingers of actuators (III) and (IV). In this design, four fixed-guided beams are designed 


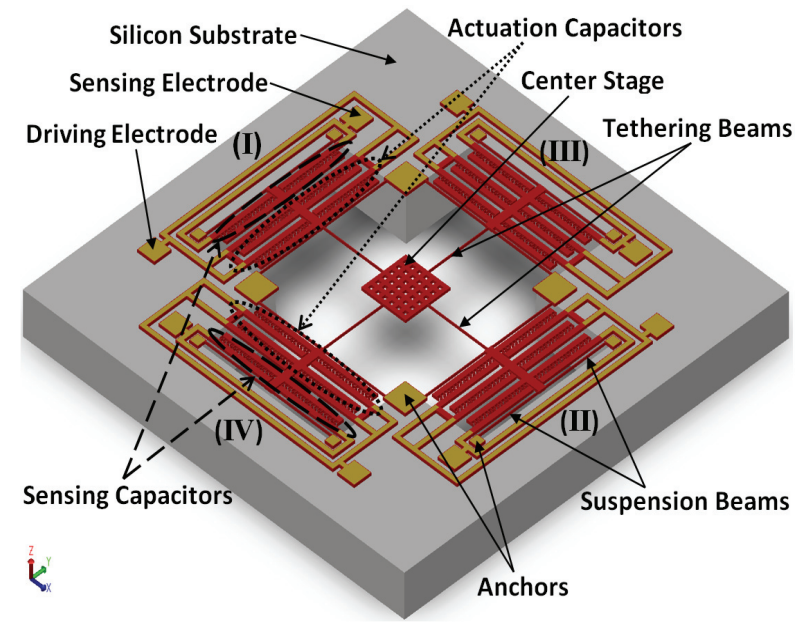

Fig. 1. Schematic diagram of the micromachined 2DOF nanopositioner.

to suspend each comb-drive actuator.

To determine device parameters, in-plane stiffness of the 2DOF nanopositioner is analyzed, and the relationships between actuation voltages and displacements in the $X Y$ directions are derived. In the analysis, it is assumed that, under small deformations, the stiffness of beams in one direction is not significantly affected by the structural deformations along other directions.

Neglecting the high lateral stiffness of the suspension beams, according to symmetry, the in-plane stiffness of the nanopositioner is [12][13]:

$$
K_{x, y}=\frac{8 E h_{s} w_{s}^{3}}{l_{s}^{3}}+\frac{2 E H_{t} W_{t}^{3}}{L_{t}^{3}}
$$

where $E=129.5 \mathrm{GPa}$ is Young's modulus of silicon, $l_{s}, w_{s}$, and $h_{s}$ are the length, width and thickness of the suspension beams, and $L_{t}, W_{t}$, and $H_{t}$ are the length, width, and thickness of the tethering beams.

The center stage is actuated by applying the driving voltages to the driving electrodes along the $X$ direction with differential $\mathrm{AC}$ voltages $\left( \pm V_{A C}\right)$ superimposed on $\mathrm{DC}$ bias $\left(V_{D C}\right)$. Since the driving voltages applied to electrodes which are placed on either side of the $X$-directional actuators are $V_{1}=$ $V_{D C}+V_{A C}$ and $V_{2}=V_{D C}-V_{A C}$ respectively (where $V_{A C}=$ $V_{d} \cdot \sin \omega t$ ), the driving force in the $X$ direction is the subtraction of the electrostatic forces [13]:

$$
F=\frac{1}{2} \frac{\partial C}{\partial x} V_{1}^{2}-\frac{1}{2} \frac{\partial C}{\partial x} V_{2}^{2}=2 \frac{\partial C}{\partial x} V_{D C} V_{A C}=\frac{4 N_{f} \varepsilon_{0} h}{d} V_{D C} V_{A C}
$$

where $\varepsilon_{0}=8.85 \times 10^{-12} C^{2} /\left(\mathrm{Nm}^{2}\right)$ is the permittivity of air, $d, h$ and $N_{f}$ are the gap, thickness and the number of comb fingers, respectively. Therefore, the in-plane displacements are [13]:

$$
x, y= \pm \frac{F}{K_{x, y}}
$$

where the $+/-$ signs represent forward and backward motions. Based on (3), the design parameters of the 2DOF nanopositioner are determined and summarized in Table 1.

TABLE I. DESIGN PARAMETERS OF THE 2DOF NANOPOSITIONER.

\begin{tabular}{|c|c|}
\hline \multicolumn{2}{|c|}{ Structural parameters } \\
\hline Suspension beams & $l_{s}=700 \mu \mathrm{m}, w_{s}=6 \mu \mathrm{m}, h_{s}=25 \mu \mathrm{m}$ \\
\hline Tethering beams & $L_{t}=700 \mu \mathrm{m}, W_{t}=6 \mu \mathrm{m}, H_{t}=25 \mu \mathrm{m}$ \\
\hline Center stage & $520 \mu \mathrm{m} \times 520 \mu \mathrm{m}$ \\
\hline Center shaft & $l_{s h}=640 \mu \mathrm{m}, w_{s h}=80 \mu \mathrm{m}$ \\
\hline Device size & $3.75 \mathrm{~mm} \times 3.75 \mathrm{~mm}$ \\
\hline \multicolumn{2}{|c|}{ Actuation and sensing parameters } \\
\hline $\begin{array}{c}\text { Comb-drive actuator } \\
\text { (one set) }\end{array}$ & $\begin{array}{c}N_{f}=256, l_{f}=30 \mu \mathrm{m}, w_{f}=3 \mu \mathrm{m}, h_{f}=25 \mu \mathrm{m}, \\
d_{f}=2 \mu \mathrm{m}, \text { overlap }_{f}=10 \mu \mathrm{m}\end{array}$ \\
\hline $\begin{array}{c}\text { Comb-drive sensor } \\
\text { (one set) }\end{array}$ & $\begin{array}{c}N_{f}=128, l_{f}=30 \mu \mathrm{m}, w_{f}=3 \mu \mathrm{m}, h_{f}=25 \mu \mathrm{m}, \\
d_{f}=2 \mu \mathrm{m}, \text { overlap }_{f}=10 \mu \mathrm{m}\end{array}$ \\
\hline \multicolumn{2}{|c|}{} \\
\hline
\end{tabular}

\section{FABRICATION}

The device was fabricated using the silicon-on-insulator (SOI) multi-user MEMS processes (SOIMUMPs) in a commercial foundry (MEMSCAP). The SOIMUMPs process is a 4-mask level SOI patterning and etching process, which offers a $25 \mu \mathrm{m}$ thick device layer and a $2 \mu \mathrm{m}$ thick buried oxide (BOX) layer supported on a $400 \mu \mathrm{m}$ thick handle layer, and a minimum gap of $2 \mu \mathrm{m}$ [14]. The fabrication process, as illustrated in Fig. 2 [15], is briefly described as follows:

1) Microfabrication starts with a highly doped n-type $25 \mu \mathrm{m}$ silicon device layer, on which a metal stack consisting of $20 \mathrm{~nm} \mathrm{Cr}$ and $500 \mathrm{~nm} \mathrm{Au}$ is patterned to allow for ohmic contact through a liftoff process;

2) Silicon is lithographically patterned with the second mask level, SOI, and etched using deep reactive ion etch (DRIE) to define both the movable and anchored features of the structure;

3) A protection polyimide layer is applied to the top surface of the silicon layer;

4) A deep trench underneath the movable structure is created by etching through the substrate layer using DRIE;

5) The exposed buried oxide layer is removed using a wet HF etch;

6) The front side polyimide layer is removed by oxygen plasma, thereby allowing the movable structure to be fully released. Then, a blanket metal layer, consisting of $50 \mathrm{~nm}$ $\mathrm{Cr}$ and $600 \mathrm{~nm} \mathrm{Au}$, is deposited and patterned using a shadow masking technique. The shadow mask is removed after evaporation, leaving a patterned metal layer on the substrate for electrical contact.

The images of the whole device and the center stage taken under a Scanning Electron Microscope (SEM) are shown in Fig. 3. As depicted in Fig. 3 (b), patterned with blanket metal, the micron-arrays on top of the center stage were designed for Atomic Force Microscope (AFM) image scanning. 


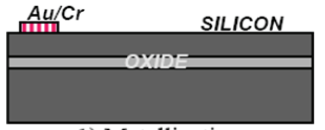

1) Metallization

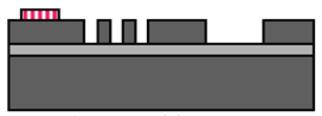

2) Front side DRIE

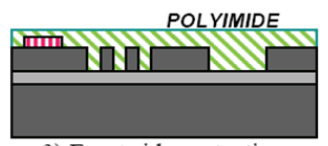

3) Front side protection

Fig. 2. Fabrication process for the 2DOF nanopositioner
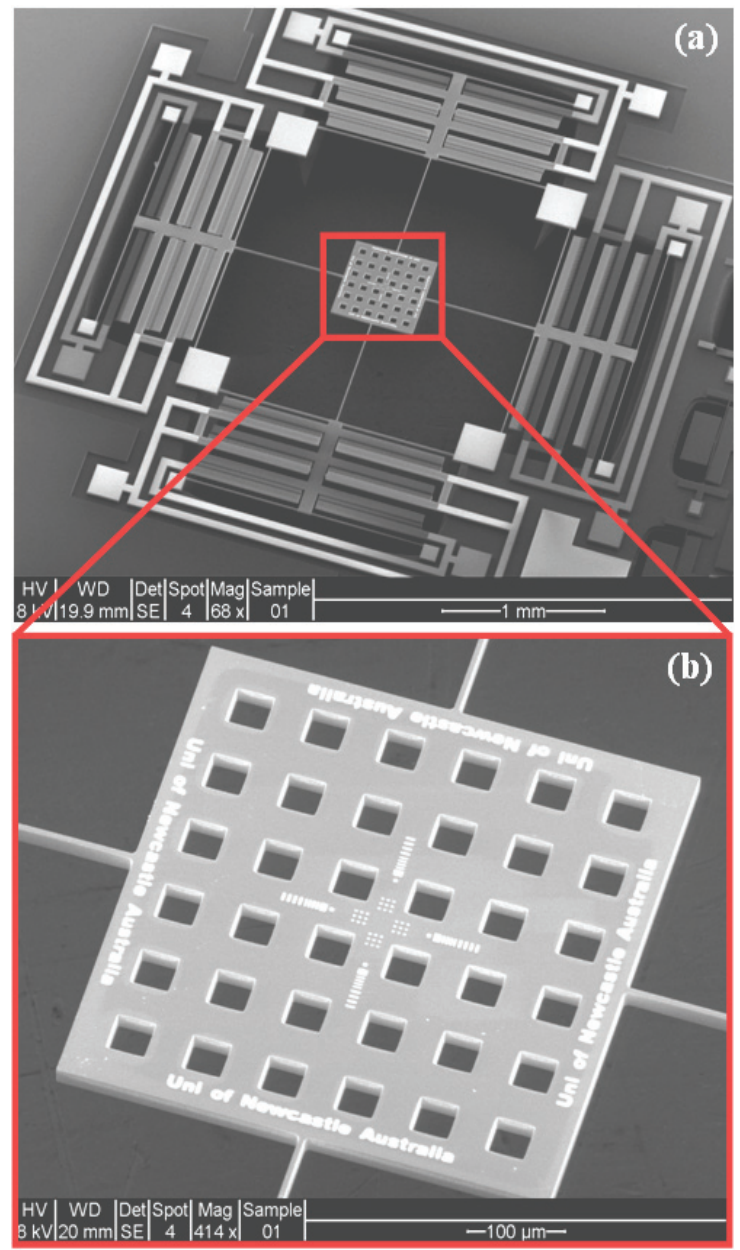

Fig. 3. SEM image of the 2DOF nanopositioner. (a) whole structure; (b) magnified view (center stage).

\section{CHARACTERIZATION}

Accurate characterization of the device is important to verify the design as well as the fabrication quality. Prior to testing, the device was glued and wire-bonded onto a PCB packaging board. The static behaviour of the nanopositioner was measured using a Polytec ${ }^{\mathrm{TM}}$ Planar Motion Analyzer (PMA). Fig. 4 shows the static bi-directional displacement of the stage along the $X$ direction against the applied DC voltage. The stage was actuated bi-directionally by applying static voltages ranging from $0 \mathrm{~V}$ to $100 \mathrm{~V}$ to the driving electrode on either side of the $X$ direction, and the displacement was measured by the PMA under different driving voltages. The stage has a dynamic range from $-6.27 \mu \mathrm{m}$ to $+6.64 \mu \mathrm{m}$ along the $X$ direction, which falls into the range of AFM scanning applications.

A capacitive readout IC (MS3110) was chosen for sensing the capacitance change and providing an output voltage proportional to that change. The advantages of MS3110 are high resolution, stability, low drift and more importantly adjustable internal balancing capacitors [16]. To minimize the stray capacitance and electromagnetic interference, the MEMS device chip is positioned very close to MS3110 chip. Fig. 5 demonstrates the experimental setup for capacitive sensing using the evaluation board MS3110BDPC [16]. Pin 4 and Pin 6 supply the capacitance bridge with AC carrier signals which are $100 \mathrm{kHz}$ square wave differential signals with a peak-to-peak amplitude of $2.25 \mathrm{~V}$. Pin 5 is kept at 2.25 $\mathrm{V}$ DC potential and connected to a common electrode that is the movable part of the MEMS structure. The feedback capacitor of the capacitance transimpedance amplifier, the output buffer gain, and the LPF bandwidth were selected to be $1.007 \mathrm{pF}, 4$, and $0.5 \mathrm{kHz}$ respectively. At every actuation voltage for the displacement measurement, the output of MS3110 (Pin 14) was measured using a digital oscilloscope (Tektronix TDS 3024B). Fig. 6 shows the measured sensor output voltage versus the stage displacement.

The dynamic behaviour of the 2DOF nanopositioner was characterized using a spectrum analyzer (HP 35670A). Superimposed on a $40 \mathrm{~V}$ DC bias, a $3 \mathrm{~V}$ sinusoidal actuation signal with different frequencies ranging from $10 \mathrm{~Hz}$ to $8 \mathrm{kHz}$ was applied to the actuator and the output signal reflecting changes in the amplitude of vibration, detected by the capacitive displacement sensor, is sent back to the spectrum analyzer. Fig. 7 presents the frequency response of the fabricated nanopositioner. The first resonance frequency is measured at $4.24 \mathrm{kHz}$, which is $15.5 \%$ higher than the simulated first undamped natural frequency of $3.67 \mathrm{kHz}$. This discrepancy is due to the fabrication imperfections. The phase delay from $10 \mathrm{~Hz}$ to $8 \mathrm{kHz}$ is $200^{\circ}$.

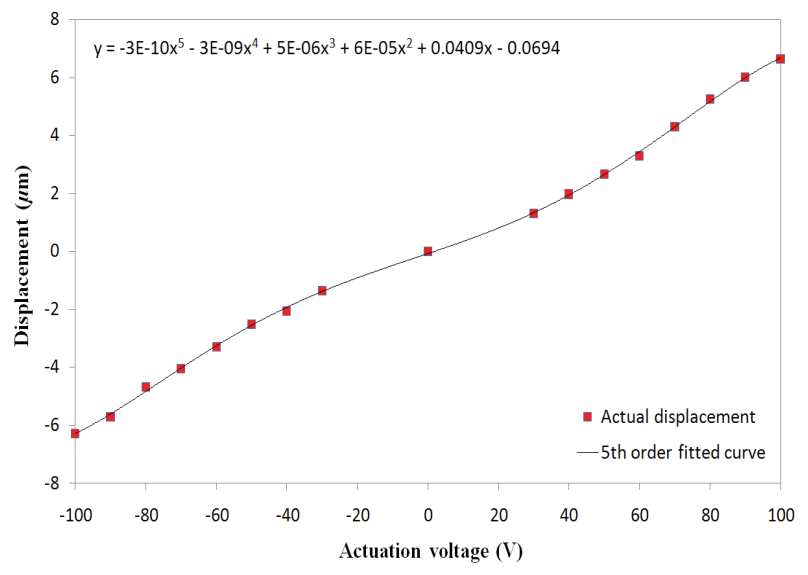

Fig. 4. Static $X$-directional displacement as a function of applied voltage. 


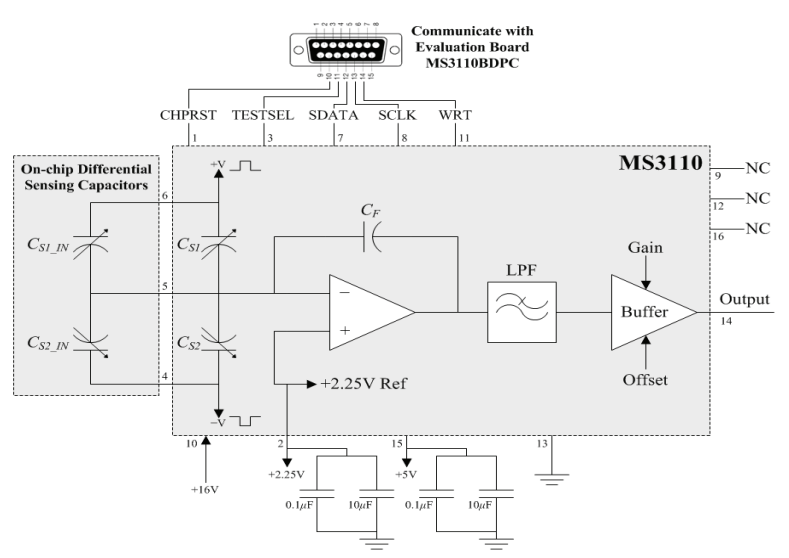

Fig. 5. Capacitive sensing measurement setup using MS3110.

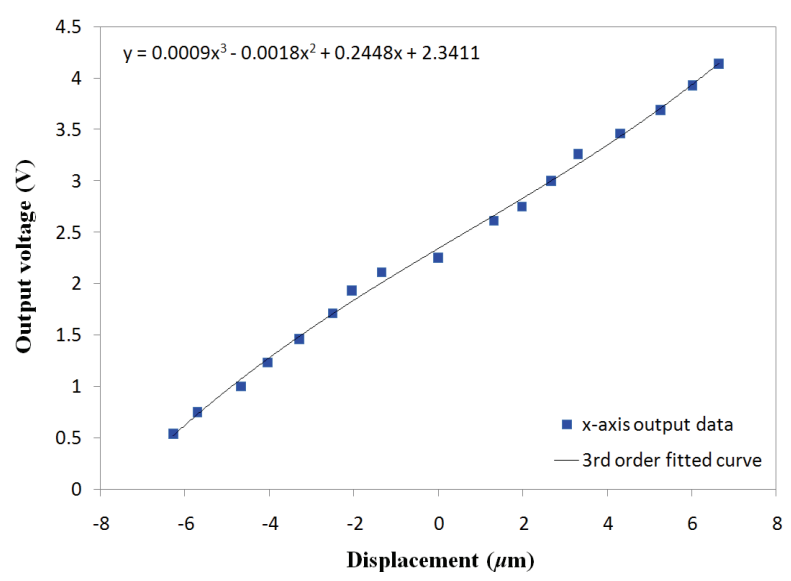

Fig. 6. Sensor output voltage as a function of $X$-directional displacement.
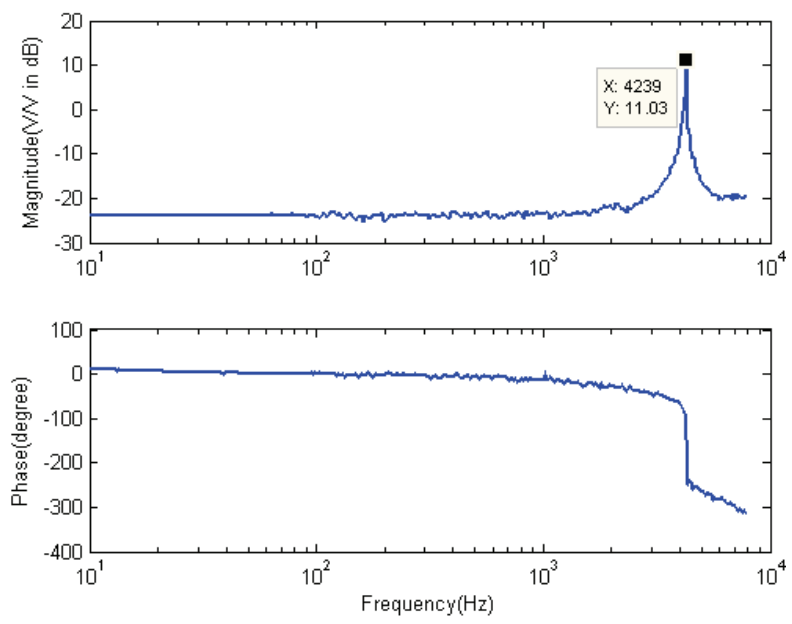

Fig. 7. Frequency response of the $X$-directional motion, both magnitude (upper trace) and phase (lower trace).

\section{CONCLUSION}

An electrostatically actuated 2DOF nanopositioner with on-chip capacitive displacement sensor is presented in this paper. With the characterization results shown in Fig. 4 and Fig. 6, a closed-loop proportional-integral (PI) controller can be designed to precisely control the developed nanopositioning stage. Furthermore, the 2-axis closed-loop feedback control of the nanopositioner will be implemented to allow for AFM imaging of the micron-arrays patterned on top of the center stage.

\section{ACKNOWLEDGMENT}

The authors would like to thank Dr. Andrew Fleming for his valuable suggestions on device packaging, and Dave Phelan from the School of Medical Science at the University of Newcastle for his help with taking SEM photographs.

\section{REFERENCES}

[1] H. Toshiyoshi, G. J. Su, J. LaCosse, and M. C. Wu, "A surface micromachined optical scanner array using photoresist lenses fabricated by a thermal reflow process," Journal of Lightwave Technology, vol. 21, no. 7, pp. 1700-1708, Jul 2003.

[2] S. H. Kwon and L. P. Lee, "Stacked two dimensional micro-lens scanner for micro confocal imaging array", in Proc. IEEE MEMS 2002, pp. 483-486, Jan 2002

[3] P. F. Indermühle, V. P. Jaecklin, J. Brugger, C. Linder, N. F. De Rooij, and M. Binggeli, "AFM imaging with an $X Y$-micropositioner with integrated TIP," Sens. Actuators A, Phys., vol. 47, no. 1, pp. 562-565, Mar 1995.

[4] C. H. Kim, H. M. Jeong, J. U. Jeon, and Y. K. Kim, "Silicon micro XY stage with a large area shuttle and no-etching holes for SPM-based data storage," J. Microelectromech. Syst., vol. 12, no. 4, pp. 470-478, Aug 2003.

[5] X. H. Huang, R. Horowitz, and Y. F. Li, "A comparative study of MEMS microactuators for use in a dual-stage servo with an instrumented suspension," IEEE/ASME Trans. Mechatronics, vol. 11, no. 5, pp. 524-532, Oct 2006.

[6] Y. K. Yong, S. S. Aphale, and S. O. R. Moheimani, "Design, identification, and control of a flexure-based XY stage for fast nanoscale positioning," IEEE Trans. Nanotechnol., vol. 8, no. 1, pp. 46-54, Jan 2009.

[7] C. L. Chu and S. H. Fan, "A novel long-travel piezoelectric-driven linear nanopositioning stage," Precis. Eng., vol. 30, no. 1, pp. 85-95, Jan 2006.

[8] D. Croft and S. Devasia, "Vibration compensation for high speed scanning tunneling microscopy," Rev. Sci. Instrum., vol. 70, no. 12, pp. 4600-4605, Dec 1999

[9] C. W. Lee and S. W. Kim, "An ultraprecision stage for alighment of wafers in advanced microlithography," Precis. Eng., vol. 21, no. 2/3, pp. 113-122, Nov 1997.

[10] S. Devasia, E. Eleftheriou, and S. O. R. Moheimani, "A survey of control issues in nanopositioning," IEEE Trans. Control Syst. Technol., vol. 15, no. 5, pp. 802-823, Sep 2007.

[11] C. Ru, B. Shao, L. Chen, W. Rong, and L. Sun, "Design, identification, and control of piezoactuated positioning mechanism based on adaptive inverse method," Proc. Inst. Mech. Eng., J. Syst. Control Eng., vol. 222 no. 16, pp. 501-509, 2008.

[12] X. Liu, K. Kim, and Y. Sun, "A MEMS stage for 3-axis nanopositioning," J. Micromech. Microeng, vol. 17, no. 9, pp. 17961802, Jul 2007.

[13] R. Legtenberg, A. W. Groeneveld, and M. Elwenspoek, "Comb-drive actuators for large displacements," J. Micromech. Microeng, vol. 6, no. 3, pp. 320-329, Jun 1996.

[14] http://www.memscap.com/en_mumps.html

[15] Y. Zhu, M. R. Yuce, and S. O. R. Moheimani, "A low-loss MEMS tunable capacitor with movable dielectric," in Proc. IEEE Sensors 2009, pp. 651-654, Oct 2009.

[16] http://www.irvine-sensors.com/pdf/MS3110\%20Datasheet\%20USE.pdf 